\title{
GLAD!
}

Revue sur le langage, le genre, les sexualités

$03 \mid 2017$

Hétérosexualités

\section{La relation entre genre grammatical et dénomination de la personne en langue française}

Résumé de thèse

The Relation between Grammatical Gender and Person Denominations in French

Lucy Michel

\section{OpenEdition}

\section{Journals}

Édition électronique

URL : http://journals.openedition.org/glad/793

DOI : $10.4000 /$ glad.793

ISSN : 2551-0819

Éditeur

Association GSL

\section{Référence électronique}

Lucy Michel, "La relation entre genre grammatical et dénomination de la personne en langue française », GLAD! [En ligne], 03 | 2017, mis en ligne le 10 décembre 2020, consulté le 20 janvier 2021. URL : http:// journals.openedition.org/glad/793; DOI : https://doi.org/10.4000/glad.793

Ce document a été généré automatiquement le 20 janvier 2021.

\section{$\circledast \oplus \Theta \Theta$}

La revue GLAD! est mise à disposition selon les termes de la Licence Creative Commons Attribution Pas d'Utilisation Commerciale - Pas de Modification 4.0 International. 


\title{
La relation entre genre grammatical et dénomination de la personne en langue française
}

\author{
Résumé de thèse \\ The Relation between Grammatical Gender and Person Denominations in French \\ Lucy Michel
}

\section{RÉFÉRENCE}

Lucy Michel. 2016. La relation entre genre grammatical et dénomination de la personne en langue française. Thèse en Linguistique, sous la direction de Philippe Monneret et Thomas Verjans, Université de Bourgogne.

1 Le point de départ de ma recherche doctorale est le constat d'une rupture dans le fonctionnement sémantico-référentiel du genre grammatical, qui oppose d'un côté noms d'inanimés et d'animés non-anthropomorphisés, et de l'autre noms d'animés humains ou anthropomorphisés. Ce constat amène inévitablement la question, souvent traitée, du type de répartition des substantifs (arbitraire ou motivé) que permet le genre grammatical (Arrivé 1997).

2 Le fait de centrer mon propos sur les noms d'humains et, plus précisément, sur la classe des «dénominations de la personne », m'a permis de sortir de cette opposition pour analyser plus précisément les difficultés posées par la catégorie grammaticale du genre dans son lien avec la bipartition sexuée des êtres humains, généralement et traditionnellement pensée comme première.

3 Cette réflexion s'appuie sur un ensemble de 496 dénominations de la personne (noms de métiers, grades, titres, mais aussi insultes) étudiées grâce à un corpus constitué de données lexicographiques, ainsi que d'extraits issus d'un ensemble de forums de discussion. 
4 À cette recherche synchronique s'ajoute un travail diachronique, visant à expliciter les mécanismes de production et de stabilisation des discours et théories sur le genre grammatical dans des ouvrages grammaticaux et lexicographiques du XVI ${ }^{\mathrm{e}}$ siècle à nos jours (Michel 2016). Ce parcours critique permet d'interroger plus précisément les normes, règles et croyances qui régissent les pratiques du genre grammatical en langue française.

5 Le travail que j'ai entrepris dans cette recherche est en grande partie fondé sur la notion de stéréotypes, ceux-ci étant entendus comme "une affaire de connaissances qu'on croit être celles qui sont l'apanage des gens en général » (Kleiber, 1999 : 73). Ce qui rend cette conception particulièrement intéressante, c'est qu'elle permet de faire le lien entre les théories de la dénomination et les outils du matérialisme et des réflexions queer sur le langage - notamment ceux développés par I. M. Young (2007) et C. Michard (1999). L'introduction d'une réflexion sur l'existence de traits pensés comme représentatifs d'un ensemble d'individus permet de questionner les processus de catégorisation dont participe le genre grammatical en langue française.

6 Ainsi, ma recherche doctorale est centrée sur une analyse stéréotypique du sens du genre grammatical : au genre grammatical masculin correspondraient un ensemble de représentations sociales de ce qu'est « le masculin », ainsi qu'un ensemble de référents dits «masculins»; au genre grammatical féminin correspondraient un ensemble de représentations sociales de ce qu'est "le féminin », ainsi qu'un ensemble de référents dits "féminins». Plutôt que les traits [+ mâle] et [+femelle], on aurait les traits [+ stéréotypes associés au groupe social femmes] et [+ stéréotypes associés au groupe social hommes].

7 Cette hypothèse permet à la fois de ne pas penser l'idée d'une hiérarchie entre les genres grammaticaux («le masculin l'emporte...») comme structurelle et interne au système linguistique français (Michel 2017), et de comprendre certains phénomènes en apparence contradictoires et généralement rejetés comme idéologiques et/ou politiques, donc non-linguistiques : questions de la féminisation, de la valeur générique du masculin, de la nature du lien entre sexe et genre grammatical, etc. L'hypothèse stéréotypique, bien que nourrie de réflexions constructivistes, n'implique cependant pas un rejet du questionnement référentiel, et émerge au contraire d'une réflexion sur le concept de catégorisation et sur les difficultés phénoménologiques et linguistiques qui y sont liées.

8 L'enjeu de cette recherche est d'abord d'intégrer à la description du genre grammatical les données socio-culturelles et historiques souvent pensées comme collatérales, mais aussi de proposer des applications possibles de cette hypothèse. Une partie de mon travail de thèse a donc été de développer un modèle d'utilisation lexicographique du sens stéréotypique du genre grammatical, visant à la fois la visibilisation des processus de construction de mots (dérivation, composition, etc.) et l'explicitation de certaines asymétries sémantiques et des stéréotypes qui les sous-tendent. 


\section{BIBLIOGRAPHIE}

ARRIVÉ, Michel. 1997. « Coup d'œil sur les conceptions du genre grammatical » Langages 124 :

113-124.

KLEIBER, Georges. 1999. La sémantique du prototype. Paris : Presses universitaires de France.

MICHARD, Claire. 1999. « Humain / femelle : deux poids deux mesures dans la catégorisation de sexe en français » Nouvelles questions féministes 20 (1), 53-95.

MICHEL, Lucy. 2016. « Penser la primauté du masculin - sémantique du genre grammatical, perspectives synchroniques et diachroniques » Actes du CMLF 2016. URL :

www.linguistiquefrancaise.org

MICHEL, Lucy. 2017. «Le "masculin culturel”, un pied de nez au "masculin générique” ? ", in Genre et sciences du langage. Enjeux et perspectives, VADOT, Maude, DAHOU, Chahrazed, ROCHE, Françoise (éd.). Montpellier : Presses universitaires de la Méditerranée, 59-78.

YOUNG, Iris Marion. 2007. «Le genre, structure sérielle : penser les femmes comme un groupe social » Recherches féministes 20, 38-61.

INDEX

Keywords : grammatical gender, denomination, semantics, stereotypes Mots-clés : genre grammatical, dénomination, sémantique, stéréotypes Thèmes : Actualités

\section{AUTEURS}

\section{LUCY MICHEL}

Lucy Michel est docteure en linguistique française et ATER à l'Université de Bourgogne. Ses travaux sont centrés sur la question du genre grammatical des dénominations de la personne en langue française, notamment d'un point de vue sémantique. Elle a soutenu une thèse sur la question en 2016. 